\title{
Mutation screening of the Ectodysplasin-A receptor gene EDAR in hypohidrotic ectodermal dysplasia
}

\author{
Annemarie H van der Hout*,1, Grétel G Oudesluijs ${ }^{1,3}$, Andrea Venema ${ }^{1}$, Joke BGM Verheij ${ }^{1}$, \\ Bart GJ Mol ${ }^{1}$, Patrick Rump ${ }^{1}$, Han G Brunner ${ }^{2}$, Yvonne J Vos ${ }^{1}$ and Anthonie J van Essen ${ }^{1}$ \\ ${ }^{1}$ Department of Genetics, University Medical Centre Groningen, University of Groningen, Groningen, The Netherlands; \\ ${ }^{2}$ Department of Human Genetics, University Medical Centre St Radboud, Nijmegen, The Netherlands
}

Hypohidrotic ectodermal dysplasia (HED) can be caused by mutations in the $\mathrm{X}$-linked ectodysplasin $\mathrm{A}$ (ED1) gene or the autosomal ectodysplasin A-receptor (EDAR) and EDAR-associated death domain (EDARADD) genes. X-linked and autosomal forms are sometimes clinically indistinguishable. For genetic counseling in families, it is therefore important to know the gene involved. In 24 of $\mathbf{4 2}$ unrelated patients with features of HED, we found a mutation in ED1. ED1-negative patients were screened for mutations in EDAR and EDARADD. We found mutations in EDAR in 5 of these 18 patients. One mutation, p.Glu354X, is novel. In EDARADD, a novel variant p.Ser93Phe, probably a neutral polymorphism, was also found. Clinically, there was a difference between autosomal dominant and autosomal recessive HED patients. The phenotype in patients with mutations in both EDAR alleles was comparable to males with X-linked HED. Patients with autosomal dominant HED had features comparable to those of female carriers of X-linked HED. The teeth of these patients were quite severely affected. Hypohidrosis and sparse hair were also evident, but less severe. This study confirms Chassaing et al's earlier finding that mutations in EDAR account for approximately $25 \%$ of non-ED1-related HED. Mutations leading to a premature stop codon have a recessive effect except when the stop codon is in the last exon. Heterozygous missense mutations in the functional domains of the gene may have a dominant-negative effect with much variation in expression. Patients with homozygous or compound heterozygous mutations in the EDAR gene have a more severe phenotype than those with a heterozygous missense, nonsense or frame-shift mutation.

European Journal of Human Genetics (2008) 16, 673-679; doi:10.1038/sj.ejhg.5202012; published online 30 January 2008

Keywords: hypohidrotic ectodermal dysplasia; mutation screening; ED1 gene; EDAR gene; EDARADD gene; genotype-phenotype correlation

\section{Introduction}

Hypohidrotic ectodermal dysplasia (HED; MIM no. 305100) is characterized by absent or a diminished number

*Correspondence: $\mathrm{Dr}$ AH van der Hout, Department of Genetics, University Medical Centre Groningen, PO Box 30 001, 9700 RB Groningen, The Netherlands.

Tel: + 3150 3617233; Fax: + 3150 3617231;

E-mail: a.h.van.der.hout@medgen.umcg.nl

${ }^{3}$ Current address: Department of Clinical Genetics, Erasmus Medical Centre, Rotterdam, The Netherlands.

Received 2 October 2007; revised 27 November 2007; accepted 20 December 2007; published online 30 January 2008 of eccrine sweat glands, missing and malformed teeth, and thin and sparse hair. The severity of symptoms can vary between and within families. The incidence is estimated to be 1 per 100000 births. ${ }^{1}$ Most cases are X-linked and are caused by mutations in the ectodysplasin A gene (ED1; MIM *300451). However, some families show autosomal dominant or autosomal recessive inheritance. Mutations in the ectodysplasin A-receptor gene (EDAR; MIM *604095) on $2 \mathrm{q} 11-13$ were found in HED families with transmission compatible with autosomal dominant and autosomal recessive inheritance, ${ }^{2-7}$ and in one consanguineous 
family with recessive HED, a homozygous mutation in the EDAR-associated death domain gene (EDARADD; MIM ${ }^{*} 606603$ ) on 1q42.2-43 was described. ${ }^{8}$ In this study, we present the results of mutation scanning of the ED1 gene in 42 HED patients, and scanning of the EDAR and EDARADD genes in ED1-negative patients. We describe the clinical features of affected individuals in five families with mutations in EDAR. In addition, we provide possible explanations for the observation (made by ourselves and others) that some mutations act in a dominant manner and others in a recessive.

\section{Materials and methods Patients}

The ED1 gene was screened in 42 unrelated index cases from The Netherlands, Belgium, Italy, Portugal, Sweden and Finland, with features suggestive for a diagnosis of HED. If no mutation was detected in ED1, the EDAR and EDARADD genes were screened. In five families (all Dutch) in which a mutation in the EDAR gene was found, patients were clinically re-evaluated. They were screened for missing or malformed teeth, and thin or sparse hair. Hydrosis was measured with an iodine sweat test in two patients. The remaining patients were questioned about their decreased sweating and heat intolerance. A written consent for use of photographs was obtained from all individuals concerned.

\section{Mutation detection}

DNA was isolated from peripheral blood leukocytes using standard laboratory methods. The ED1 gene was screened by Denaturing Gradient Gel Electrophoresis (exons 3, 4, 6, 7, 8 and 9) and direct sequencing (exons 1 and 5). Primer sequences are available upon request. All exons and flanking intronic sequences of the EDAR and EDARADD genes were amplified by PCR using previously published primers. ${ }^{2,8}$ Products were directly sequenced using an ABI 3100 Genetic Analyzer (Applied Biosystems, Foster City, CA, USA).

\section{Results}

In 24 of 42 unrelated patients with a clinical diagnosis or strong suspicion of having HED, a mutation in the ED1 gene was detected. Mutations are summarized in Table 1. In the remaining 18 patients ( 9 male, 9 female), the $E D A R$ and $E D A R A D D$ genes were screened. A pathogenic mutation in $E D A R$ was detected in five families. Table 2 summarizes our data and mutations published earlier.

A novel variant in the EDARADD gene was found in a sporadic female patient with conical teeth and sparse downy hair: c.278C $>$ T/p.Ser93Phe (A of the first ATG $\operatorname{codon}=1$, based on reference sequence NM_080738). Mutations in the ED1 and EDAR genes were excluded. This variant was also found in the mother, who shows no signs of HED.

\section{Patients with mutations in EDAR}

All the parents of affected children were unrelated and their family history was non-contributory unless otherwise stated. Intelligence and development, besides their symptoms of HED, were normal in all patients.

Family 1, patients 1 and 2 In this family, there are two affected brothers (1 and 2). At the ages of 2 years and 6 months, respectively, they were clinically examined, and

Table 1 Mutations identified in the ED1 gene in this study ${ }^{a}$

\begin{tabular}{|c|c|c|c|c|}
\hline Nucleotide change & Protein change & Location & Mutation type & No. of families \\
\hline c.1-?_396+?del & & Exon 1 & Exon deletion & 1 \\
\hline c. $2 \mathrm{~T}^{-}>\mathrm{A}$ & p.Met1Lys & Exon 1 & Loss initiation site & 1 \\
\hline c.77delG & p.Gly26fs & Exon 1 & Frameshift & 1 \\
\hline c. $463 C>T$ & p.Arg155Cys & Exon 3 & Missense & 3 \\
\hline c. $466 \mathrm{C}>\mathrm{T}$ & p.Arg156Cys & Exon 3 & Missense & 1 \\
\hline c. $467 G>A$ & p.Arg156His & Exon 3 & Missense & 3 \\
\hline c.546_581del36 & p.Gly183_Pro194del & Exon 5 & In-frame deletion & 1 \\
\hline c.553_588del36 & p.Asn185_Pro196del & Exon 5 & In-frame deletion & 1 \\
\hline c.559_576del18 & p.Pro184_Gly189del & Exon 5 & In-frame deletion & 1 \\
\hline$c .741+1 G>T$ & & Intron 6 & Splice & 1 \\
\hline c. $793+2 T>C$ & & Intron 7 & Splice & 1 \\
\hline c.795_796insTTAT & p.Gly268fs & Exon 8 & Frameshift & 1 \\
\hline c. $871 \bar{G}>A$ & p.Gly291 Arg & Exon 8 & Missense & 1 \\
\hline c. $892 \mathrm{G}>\mathrm{T}$ & p.Asp298Tyr & Exon 8 & Missense & 1 \\
\hline c.920T $>G$ & p.Val307Gly & Exon 9 & Missense & 1 \\
\hline c. $924+8 C>A$ & & Intron 8 & Splice & 1 \\
\hline c. $925-3 C>G$ & & Intron 8 & Splice & 1 \\
\hline c. $1114 A>G$ & p.Asn372Asp & Exon 9 & Missense & 1 \\
\hline C. $1119 G>A$ & p.Met373Ile & Exon 9 & Missense & 1 \\
\hline c. 1061 1110del50 & p.Leu354fs & Exon 9 & Frameshift & 1 \\
\hline
\end{tabular}

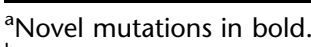

${ }^{b}$ Mutation nomenclature is according to reference sequence NT_011669.16 with numbering starting at the A of the first ATG. 
Table 2 Summary of known mutations in the EDAR gene

\begin{tabular}{|c|c|c|c|c|c|c|}
\hline $\begin{array}{l}\text { Nucleotide } \\
\text { change }^{\mathrm{a}}\end{array}$ & Protein change $e^{\mathrm{a}}$ & Location & $\begin{array}{l}\text { Mutation } \\
\text { type }\end{array}$ & Inheritance & $\begin{array}{l}\text { Family no. } \\
\text { this study }\end{array}$ & Reference \\
\hline c.52-25_52-8del & & Intron 2 & Splice & $A R$ & & Monreal et $a l^{2}$ \\
\hline c. $51+1 \bar{G}>A$ & & Intron 2 & Splice & $A R$ & & Shimomura et $\left.a\right|^{5}$ \\
\hline C. $140 G>A$ & p.Cys47Tyr & Exon 3 & Missense & $A R$ & & Chassaing et $a P^{3}$ \\
\hline c. $259 \mathrm{~T}>\mathrm{C}$ & p.Cys87Arg & Exon 4 & Missense & $A R$ & & Monreal et $a l^{2}$ \\
\hline c. $266 \mathrm{G}>\mathrm{A}$ & p.Arg89His & Exon 4 & Missense & $\begin{array}{l}A R \\
A D\end{array}$ & 1 & $\begin{array}{l}\text { Monreal et } a l^{2} \text { Chassaing et } a l^{3} \\
\text { This study }\end{array}$ \\
\hline > exon 4 del & & & Large deletion & $A R$ & & Monreal et $a l^{2}$ \\
\hline c. $329 \mathrm{~A}>\mathrm{C}$ & p.Asp110Ala & Exon 4 & Missense & $A R$ & 1 & Chassaing et $a l^{3}$ and this study \\
\hline c.399_404del6 & $\begin{array}{l}\text { p.Met133 } \\
\text { Cys135delinslle }\end{array}$ & Exon 5 & In-frame deletion & $A R$ & & Tariq et ap \\
\hline c. $442 \mathrm{~T}>\mathrm{C}$ & p.Cys148Arg & Exon 5 & Missense & $A R$ & & Chassaing et $a l^{3}$ \\
\hline c. $528+1 \mathrm{G}>\mathrm{A}$ & & Intron 6 & Splice & AR & & Chassaing et $a l^{3}$ \\
\hline c.718_721del & p.Lys740fs & Exon 8 & Frameshift & AR & & Naeem et $a t^{4}$ \\
\hline c. $106 \overline{0} \mathrm{G}>\mathrm{T}$ & p.Glu354X & Exon 12 & Nonsense & $A D$ & 2 & This study \\
\hline c. $1072 \mathrm{C}>\mathrm{T}$ & p.Arg358X & Exon 12 & Nonsense & $A D$ & 3,4 & $\begin{array}{l}\text { Monreal et al, }{ }^{2} \text { Lind et } a l_{,}{ }^{6} \\
\text { and this study }\end{array}$ \\
\hline c. $1124 G>A$ & p.Arg375His & Exon 12 & Missense & AR & & Shimomura et $a P^{5}$ \\
\hline C. $1129 \mathrm{C}>\mathrm{T}$ & p.Leu377Phe & Exon 12 & Missense & $A D$ & & Chassaing et $a P^{3}$ \\
\hline c. $1144 G>A$ & p.Gly382Ser & Exon 12 & Missense & $A R$ & & Naeem et al ${ }^{4}$ \\
\hline c. $1208 \mathrm{C}>\mathrm{T}$ & p.Thr403Met & Exon 12 & Missense & AR & & Chassaing et $a l^{3}$ \\
\hline c. $1237 A>C$ & p.Thr413Pro & Exon 12 & Missense & $A D$ & & Chassaing et $a l^{3}$ \\
\hline c. $1253 \mathrm{~T}>\mathrm{C}$ & p.lle418Thr & Exon 12 & Missense & AD? & & Chassaing et $a \beta^{3}$ \\
\hline c. $1259 \mathrm{G}>\mathrm{A}$ & p.Arg420Gln & Exon 12 & Missense & $A D$ & 5 & $\begin{array}{l}\text { Monreal et } a l^{2} \text { Chassaing et } a l^{3} \\
\text { and this study }\end{array}$ \\
\hline c. $1302 \mathrm{G}>\mathrm{T}$ & p.Trp434Cys & Exon 12 & Missense & AR & & Chassaing et al \\
\hline
\end{tabular}

$A D$, autosomal dominant; $A R$, autosomal recessive.

${ }^{a}$ Mutation nomenclature is according to reference sequence NT_022171 with numbering starting at the A of the first ATG.

again 18 months later. They both had conical teeth and hypodontia, thin and sparse hair, and decreased sweating, comparable to boys with X-linked recessive HED. An X-ray revealed that the oldest boy has only two permanent teeth. He has recurrent atrophic rhinitis, and has had multiple respiratory infections. The father has no features of HED, while the mother has only some mild features. She had hypohidrosis and only a few permanent teeth, with milk teeth that had remained. Two EDAR mutations, p.Arg89His and p.Asp110Ala, were found in both the affected boys. The unaffected father was a carrier of the p.Asp110Ala mutation and the mother of the p.Arg89His mutation.

Family 2, patients 3 and 4 Patient 3, a boy aged 18 months is the only child of these parents. The patient's history mentioned that his sweating was decreased. When the weather is warm, he has syncopal episodes and tries to crawl into the refrigerator. He had thin, brittle, curly, scalp hair, sparse eyebrows and eyelashes, retrognathia, and prominent ears. There were two small conical teeth in the maxilla (Figures 1c and d). The nails had longitudinal grooves and the skin was dry and eczematous. He was $82 \mathrm{~cm}$ tall $(<0 \mathrm{SD})$, weighed $9.4 \mathrm{~kg}(<2 \mathrm{SD})$ and his OFC was $45.3 \mathrm{~cm}(2 \mathrm{SD})$.

His father has no features of HED but his mother's case history (patient 4) mentioned delayed eruption of milk teeth, although, unfortunately, no exact data could be given. The mandibular and maxillary cuspids and first bicuspids of her permanent teeth had not erupted (Figures $1 \mathrm{a}$ and b). Sweating was also decreased. Clinical examination of the mother showed sparse and thin, brittle scalp hair, sparse eyebrows and eyelashes. All her maxillar teeth were partial dentures, and her mandibular cuspids and first bicuspids were absent. Her mammary glands were underdeveloped and areolae were hypoplastic. The skin was dry and eczematous. Axillary and pubic hair was sparse. The mother's family history was inconclusive for features of HED. One allele in the mother had a p.Glu354X mutation; her son (3) was not tested.

Family 3, patients 5 and 6 In this family, the female index patient 5 had one brother and two sisters; her eldest sister had died shortly before birth (probably due to an intrauterine infection). The girl's case history mentioned slightly decreased sweating and a mild sensorineural hearing loss of $10-20$ and $20-40 \mathrm{~dB}$ in the right and left ears, respectively. Clinical evaluation of the girl at 13 years of age showed thin and sparse scalp hair, sparse eyebrows and eyelashes, hypoplastic alae nasi, thin upper lip, maxillary hypoplasia, and a high frontal hairline (Figures $2 \mathrm{c}$ and d). Several teeth were missing and only two maxillary and mandibular incisors were present. There were no nail or skin abnormalities. She was $147.5 \mathrm{~cm}$ tall ( $-2 \mathrm{SD})$, weighed $31.2 \mathrm{~kg}(-2 \mathrm{SD})$, and her OFC was $52 \mathrm{~cm}(-1 \mathrm{SD})$.

Her father had no features of HED but her mother's history (patient 6) mentioned decreased sweating and that 

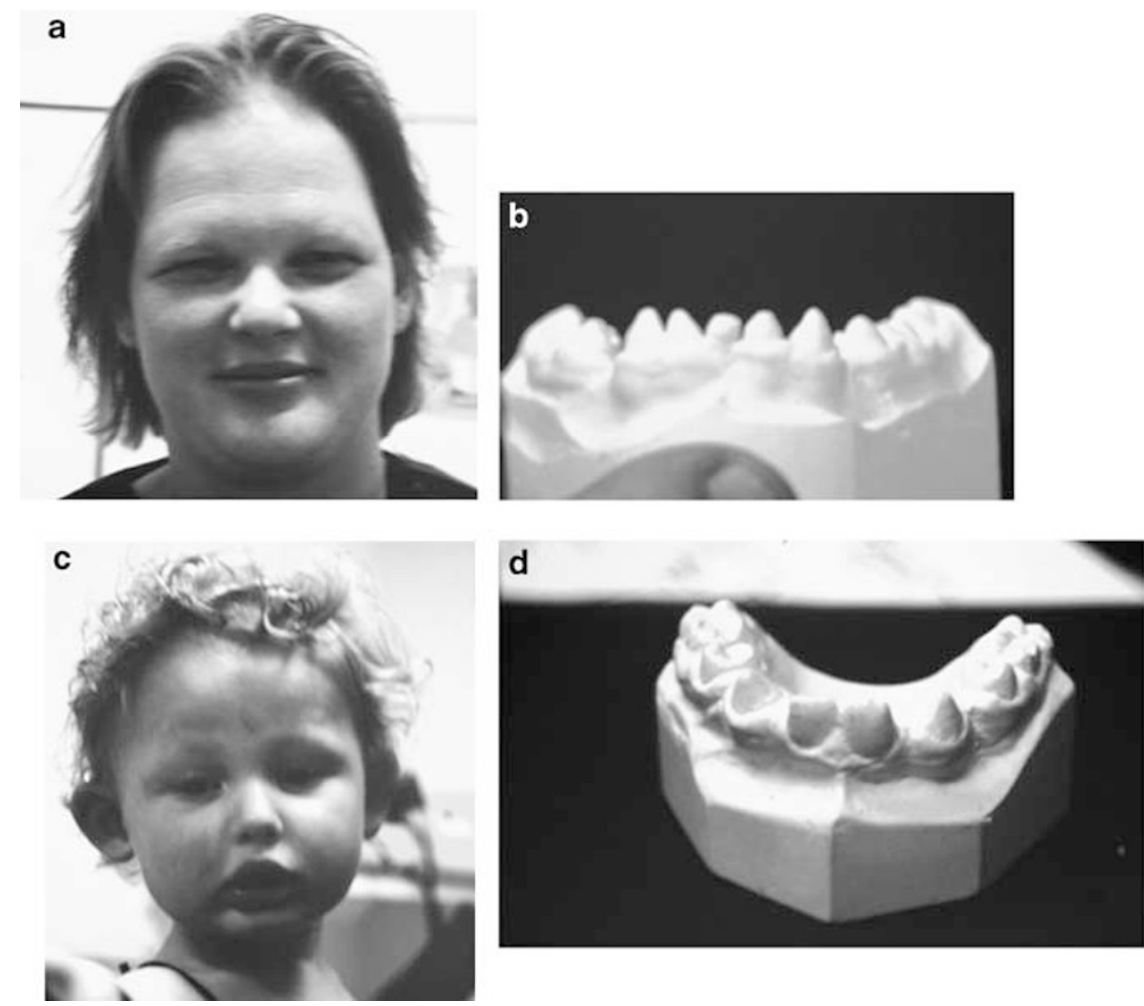

Figure 1 Patient 4 (a and $\mathbf{b}$ ) and patient 3 (c and $\mathbf{d}$ ) of family 2. Note the sparse scalp hair and oligodontia and conical teeth.

all permanent teeth had failed to erupt. Clinical investigation showed sparse scalp hair, eyebrows and eyelashes, hypoplastic alae nasi, thin upper lip, and a high frontal hairline (Figures 2a and b). Maxillar and mandibular dentures had been placed. Pubic hair was normal but axillary hair was patchy. Her nails and skin were normal. There is no information about her parents. However, her sister and her nephew from this sister have similar clinical features, although the boy has less severe symptoms. His hair is fairly full, but several of his permanent teeth are missing.

A heterozygous EDAR mutation p.Arg358X was identified in both the index case (5) and her mother (6).

Family 4, patients 7 and 8 The female index patient 7 in family 4 has one brother and one sister. Her case history mentioned decreased sweating and failure of some of her permanent teeth to erupt, although the permanent maxillary central incisors, and two maxillar and mandibular molars bilateral had erupted. The girl was clinically examined at age 9.5 years. She had sparse scalp hair, eyebrows and eyelashes (Figures $3 c-e$ ). There were no nail abnormalities, but her skin was dry and eczematous, and she had five café au lait spots $(>1 \mathrm{~cm})$ on her trunk. Nipples and areolae were normal. An iodine sweat test showed diffuse hypohidrosis. She was $144 \mathrm{~cm}$ tall $(0 /+1$ SD). Her father had no features of HED but her mother's case history (patient 8) mentioned insufficient breast milk, decreased sweating and heat intolerance. Some of the mother's teeth had failed to erupt: only bilateral two molars maxillar and mandibular, one conical cuspid mandibular, and four conical incisors maxillar had erupted. Clinical examination of the mother showed sparse scalp hair, eyebrows and eyelashes (Figures $3 a$ and b). Her pubic hair was normal, but axillary hair was sparse; her nails and skin were normal, as were her mammary glands, nipples and areolae. An iodine sweat test showed diffuse hypohidrosis. A heterozygous de novo EDAR mutation p.Arg358X was found in the mother who passed it on to her daughter. The mother's parents were unaffected and did not carry this mutation.

Family 5, patients 9 and 10 The male index patient 9 has one younger, unaffected sister. He was clinical examined at 16 months of age. He had frontal Bossing and retrognathia, thin, sparse scalp hair and eyebrows; and there were two conical teeth in the maxilla. His nails were normal, and his skin was dry and eczematous. He was $88.7 \mathrm{~cm}$ tall $(+2 \mathrm{SD})$. His father had no features of HED but his mother's case history (patient 10) mentioned decreased sweating and a reduced number of milk and permanent teeth. Only a bilateral cuspid maxillar and mandibular, one molar mandibular at the left, and a bilateral molar maxillar and mandibular had erupted. The maxillary teeth were 

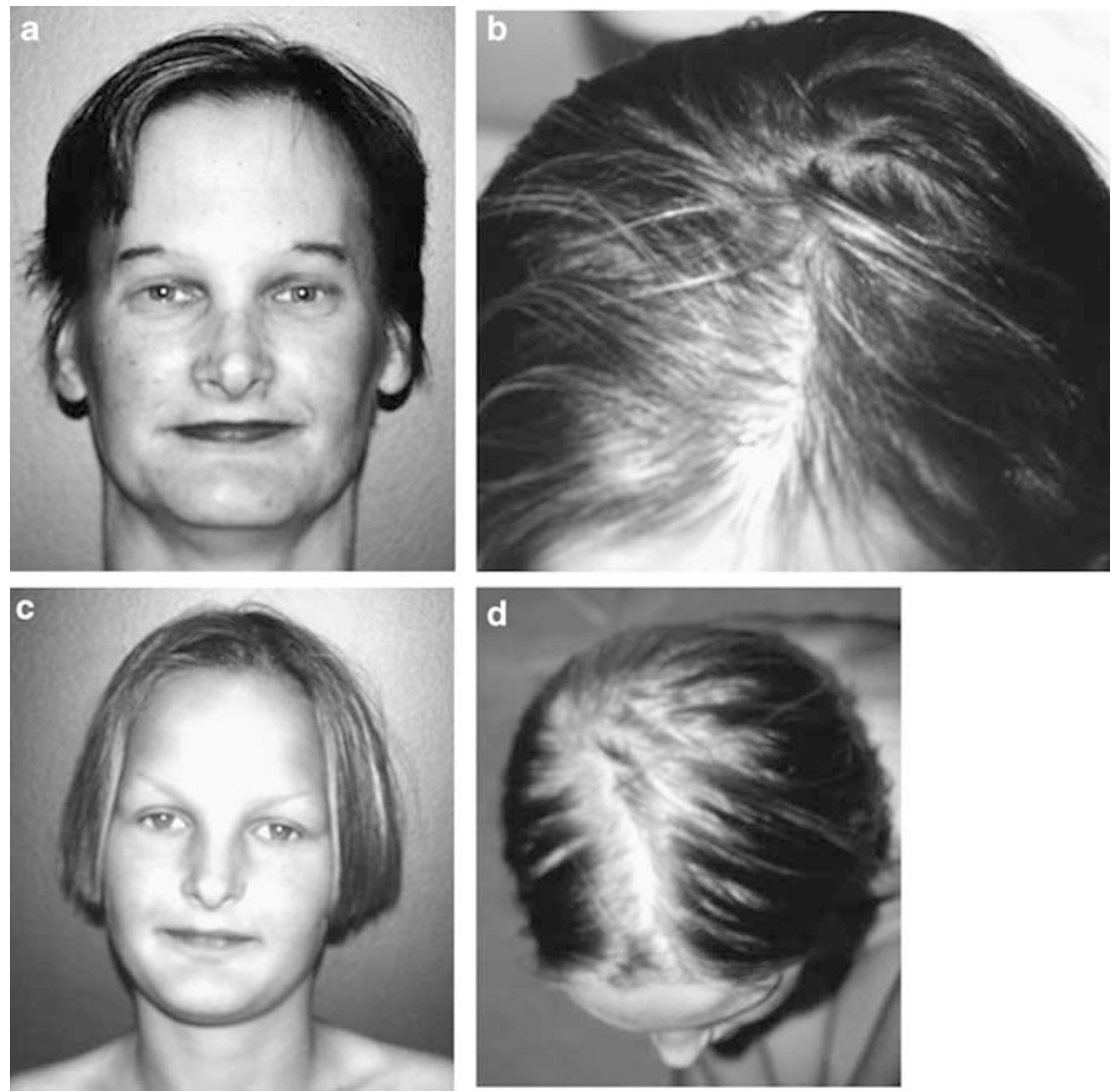

Figure 2 Patient 6 ( $\mathbf{a}$ and $\mathbf{b}$ ) and patient 5 (c and $\mathbf{d}$ ) of family 3. Note the sparse scalp hair and eyebrows, hypoplastic alae nasi, maxillary hypoplasia, and high frontal hairline. Eyebrows of patient 6 have been dyed.

conical. Clinical examination of the mother showed sparse scalp hair and eyebrows, normal nails, and dry skin. Mandibular and maxillar dentures had been placed.

The mother's family history was positive for features of HED: her brother had sparse hair, teeth abnormalities and eczema. The maternal grandmother of the male index patient and her sister also have sparse hair, teeth abnormalities, and eczema. At least nine other family members have symptoms consistent with HED. The index patient 9 was not tested, but his mother (10) carries an EDAR mutation p.Arg $420 \mathrm{Gln}$.

\section{Discussion}

\section{Mutation screening in the ED1 gene}

In 24 out of $42(57 \%)$ of the suspected HED families, all with a male index case, we detected a mutation in the ED1 gene. There are 20 different mutations, 10 of these are novel. The spectrum of mutations is comparable to series published before. $^{9}$

\section{Mutation screening in the $E D A R$ gene}

Five of the $18 E D 1$-negative index patients (28\%) had mutations in the EDAR gene. In four of these families, the pedigree was compatible with an autosomal dominant inheritance pattern, and in three of them, we found mutations already described as causing autosomal dominant HED: p.Arg358X in families 3 and 4, and p.Arg420Gln in family $5 .^{2,3,6}$ In the fourth dominant family (family 2), a novel mutation, p.Glu354X, was found. The inheritance pattern in one family (family 1) was less clear: the two severely affected brothers were compound heterozygous for two separate mutations, p.Arg89His and p.Asp110Ala, their asymptomatic father carries the p.Asp110Ala mutation, whereas the mildly affected mother carries the p.Arg89His mutation. Sequencing of the complete EDAR gene in the mother revealed no other mutations. Both mutations have been described in combination with other mutations in trans, in families showing autosomal recessive inheritance. ${ }^{2,3}$ However, these reports do not mention whether the carrier parents had been extensively clinically evaluated. Subtle abnormalities, as in the mother of these two boys, may have been missed. The severity of the phenotype in the brothers is in favor of two inactive alleles. Thus, a seemingly recessive mutation can also give rise to phenotypic expression in a carrier.

So far 20 HED families, carrying 20 different EDAR mutations, have been described in the literature. ${ }^{2-7}$ 
a
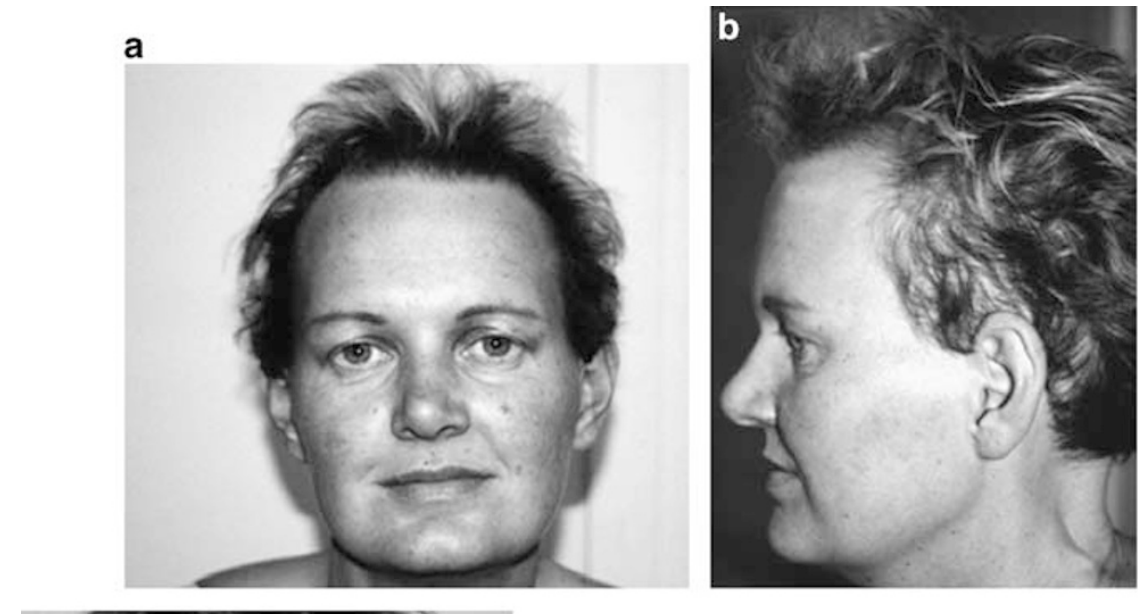
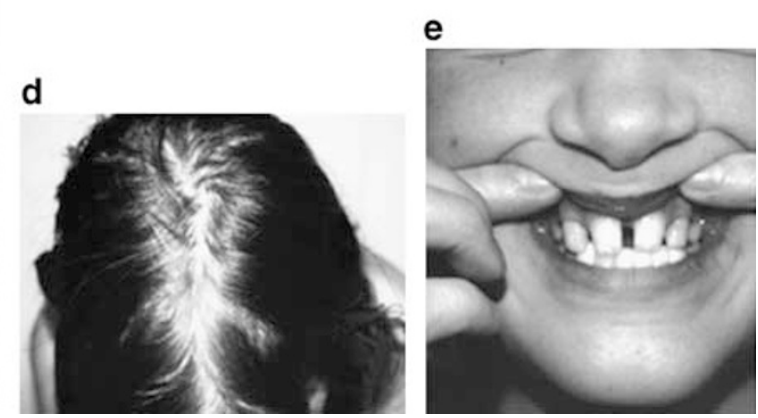

Figure 3 Patient 8 (a and $\mathbf{b}$ ) and patient 7 (c, $\mathbf{d}$ and $\mathbf{e}$ ) of family 4 . Note the sparse scalp hair and eyebrows, and missing teeth. Make up has been applied to the eyebrows of patient 8 .

Chassaing $\mathrm{et}^{3}$ found an EDAR mutation in $9 / 37$ (24\%) of their ED1-negative cases, compared to 5/18 (28\%) in our population. Thus, our study confirms their finding that mutations in $E D A R$ account for approximately $25 \%$ of nonED1-related HED cases.

For genetic counseling purposes, it is important to predict the mode of inheritance for any novel mutation found. A correlation between the location and nature of the mutations and the mode of inheritance is now emerging. Mutations leading to a premature stop codon in the mRNA have a recessive effect, because the mutant mRNA is expected to be degraded by the nonsensemediated mRNA decay (NMD) pathway. Only mutations causing premature stop codons in the last exon, such as p.Glu354X and p.Arg358X, do not cause NMD, because this is depending on an upstream exon-exon junction (for a review of NMD see Maquat ${ }^{10}$ ). The truncated protein products formed by these mutants, missing the death domain (DD), can have a dominant-negative effect on the protein function, presumably due to lack of homotrimerization of the DDs.

All missense mutations are located in the functional ligand binding domain or the DD, except p.Trp434Cys, which is located shortly after the DD. In most cases, the mode of inheritance of these mutations seems to be recessive, although the mother in our family 1 , who is a heterozygous carrier of p.Arg89His, shows very mild signs of HED. Chassaing et al described an affected girl and her very mildly affected father who both carry p.Ile418Thr. We suspect all missense mutations may have a dominant effect with extensive phenotypic variability. The phenotype is more severe if a mutation is present on both alleles. Further examination of the carrier relatives of patients with apparently recessive disease could yield support for this hypothesis.

In the European/American population, there are several recurrent mutations, p.Arg89His, p.Asp110Ala, p.Arg358X, and p.Arg420Gln. Of the six mutations found in our study of 42 Dutch patients, only one (p.Glu354X) is novel (Table 2). In contrast, in four Asian families, five mutations have been described, all exclusive for one family. ${ }^{4,5,7}$

\section{Mutation screening in the EDARADD gene}

In one of our patients, we found a variant p.Ser93Phe. This novel variant is most likely a neutral polymorphism and 
not a pathogenic mutation. Serine 93 is not evolutionary conserved between human and mouse, whereas there is more than $75 \%$ homology between human and murine $E D A R A D D .^{8}$ This amino-acid residue is not located in the functionally important DD. p.Ser93Phe is unlikely to be a dominant mutation because the mother of this patient is also a carrier of this variation, but she has no symptoms of HED.

\section{Phenotype}

Phenotypically the autosomal dominant HED families show a relatively mild phenotype in comparison to autosomal recessive and X-linked families. In most patients, the teeth are quite severely affected (missing or malformed), in contrast to the relatively mild hypohidrosis and hypotrichosis seen in some patients. These last two features might have been missed in other studies if an extensive clinical evaluation was not performed. There is a wide intrafamilial and interfamilial variability.

\section{Acknowledgements}

We thank the patients and their families for their kind cooperation. Clinicians are acknowledged for sending DNA samples from their HED patients to our laboratory. We thank Mrs Jackie Senior for critically reviewing the paper.

\section{References}

1 Stevenson AC, Kerr CB: On the distribution of frequencies of mutation to genes determining harmful traits in man. Mutat Res 1967; 4: 339-352.

2 Monreal AW, Ferguson BM, Headon DJ, Street SL, Overbeek PA, Zonana J: Mutations in the human homologue of mouse dl cause autosomal recessive and dominant hypohidrotic ectodermal dysplasia. Nat Genet 1999; 22: 366-369.

3 Chassaing N, Bourthoumieu S, Cossee M, Calvas P, Vincent MC: Mutations in EDAR account for one-quarter of non-ED1-related hypohidrotic ectodermal dysplasia. Hum Mutat 2006; 27: 255-259.

4 Naeem M, Muhammad D, Ahmad W: Novel mutations in the EDAR gene in two Pakistani consanguineous families with autosomal recessive hypohidrotic ectodermal dysplasia. $\mathrm{Br} J$ Dermatol 2005; 53: 46-50.

5 Shimomura Y, Sato N, Miyashita A, Hashimoto T, Ito M, Kuwano R: A rare case of hypohidrotic ectodermal dysplasia caused by compound heterozygous mutations in the EDAR gene. I Invest Dermatol 2004; 123: 649-655.

6 Lind KL, Stecksén-Blicks C, Lejon K, Schmitt-Egenolf M: EDAR mutation in autosomal dominant hypohidrotic ectodermal dysplasia in two Swedish families. BMC Med Genet 2006; 7: 80.

7 Tariq M, Wasif N, Ahmad W: A novel deletion mutation in the EDAR gene in a Pakistani family with autosomal recessive hypohidrotic ectodermal dysplasia. Br J Dermatol 2007; 157: 207-209.

8 Headon DJ, Emmal SA, Ferguson BM et al: Gene defect in ectodermal dysplasia implicates a death domain adapter in development. Nature 2001; 414: 913-916.

9 Vincent MC, Biancalana V, Ginisty D, Mandel JL, Calvas P: Mutational spectrum of the ED1 gene in X-linked hypohidrotic ectodermal dysplasia. Eur J Hum Genet 2001; 9: 355-363.

10 Maquat LE: Nonsense-mediated mRNA decay in mammals. J Cell Sci 2005; 118: 1773-1776. 